\title{
Predictors of Surgery and Cost of Care Associated with Patellar Instability in the Pediatric and Young Adult Population
}

\author{
Lambert T. Li, B.A., Steven L. Bokshan, M.D., Nicholas J. Lemme, M.D., \\ Edward J. Testa, M.D., Brett D. Owens, M.D., and Aristides I. Cruz Jr., M.D., M.B.A.
}

\begin{abstract}
Purpose: To determine how patient demographics, socioeconomic status, history of recurrence, and initial point of presentation for health care influenced the decision for surgical treatment following a patellar instability episode. Methods: The New York SPARCS database from 2016 to 2018 was queried for patients aged 21 and younger who were diagnosed with a patellar instability episode. These were linked to later surgeries with Current Procedural Terminology (CPT) codes 27405 (MPFL repair), 27418 (tibial tubercle osteotomy), 27420 (dislocating patella reconstruction), 27422 (Campbell/Roux-Goldthwait procedure), and 27427 (extra-articular knee ligamentous reconstruction). $\chi^{2}$-analysis and binary logistic regression were used to assess demographic and injury-specific variables for association with operative management. A generalized linear model was used to estimate charges associated with patellar instability. Results: There were 2,557 patients with patellar instability, $134(5.2 \%)$ of whom underwent surgery. Patients with recurrent instability had 1.875 times higher odds of undergoing surgery $(P=.017)$. Compared to white patients, black patients had 0.428 times the odds of surgery $(P=.004)$. None of the patients without insurance had surgery. In the cost model, an initial visit to an outpatient office was associated with \$1,994 lower charges compared to an emergency department (ED) visit $(P<.001)$. Black patients had $\$ 566$ more in charges than White patients $(P=.009)$. Compared with nonoperative treatment, surgeries with CPT 27405 added \$13,124, CPT 27418 added \$10,749, CPT 27422 added \$18,981, CPT 27420 added \$23,700, and CPT 27427 added $\$ 25,032$ (all $P<.001$ ). Conclusions: Patients with recurrent instability had higher odds of surgery, while Black and uninsured patients had lower odds of surgery. ED visits were associated with significantly higher charges compared to office visits, and Black patients had higher charges than white patients. Minority and uninsured patients may face barriers in access to orthopedic care. Level of Evidence: Level III, retrospective cohort study.
\end{abstract}

\section{Introduction}

$\mathbf{P}$ atellar instability (PI), which includes either dislocation or subluxation of the patella from the femoral trochlea, is a common injury in pediatric and adolescent patients. ${ }^{1}$ Waterman et al. demonstrated the incidence of PI to be 2.29 events per 100,000 person-years, with patients 15-19 years old having the highest risk of instability at an incidence of 11.9 per 100,000 person-years. ${ }^{2}$ For high school athletes, PI has been estimated to occur in

Department of Orthopaedic Surgery, Warren Alpert School of Medicine, Brown University, Providence, Rhode Island, U.S.A.

The authors report the following potential conflicts of interest or sources of funding: S. L. Bokshan reports personal fees from Zimmer Biomet and Stryker, outside the submitted work. B. D. Owens reports personal fees from Musculoskeletal Transplant Foundation, Linvatec, DePuy Synthes, and Vericel, outside the submitted work. A. I. Cruz reports personal fees from OrthoPediatrics Corp., outside the submitted work. N. J. Lemme reports personal fees from Stryker, outside the submitted work. Full ICMJE author disclosure forms are available for this article online, as supplementary material.
1.95 per 100,000 athletic exposures, while for collegiate athletes the incidence may be as high as 16.1 incidents per 100,000 athlete exposures. ${ }^{3,4}$ PI commonly occurs during athletic activity, and as such, with the rise in participation, intensity, and frequency of youth sports, it is prudent to properly manage patients with PI to decrease the risk of recurrence, the associated physical and psychosocial impairments, any functional deficits, and future risk of osteoarthritis. ${ }^{2,5-7}$

\footnotetext{
Received October 7, 2020; accepted May 31, 2021

Address correspondence to Lambert T. Li, B.A., Department of Orthopaedic Surgery, Sports Injury Laboratory, Brown University, Warren Alpert School of Medicine, 1 Kettle Point Ave., Providence, RI 02906, U.S.A. E-mail: Lambert_Li@brown.edu

(C) 2021 THE AUTHORS. Published by Elsevier Inc. on behalf of the Arthroscopy Association of North America. This is an open access article under the CC BY-NC-ND license (http://creativecommons.org/licenses/by-nc-nd/4.0/). 2666-061X/201623

https://doi.org/10.1016/j.asmr.2021.05.008
} 
The management of patients with patellar instability varies amongst providers, and the management of a first-time dislocation is particularly controversial. ${ }^{8,9}$ Traditionally, patients presenting with a first-time instability event without evidence of gross instability or concomitant injury, such as an osteochondral loose body, have been managed nonoperatively, which consists of a brief period of immobilization, followed by rehabilitation, and a gradual return to activity. ${ }^{5}$ Surgical intervention is typically reserved for patients with gross instability following reduction, patients with recurrent instability, and patients with concomitant osteochondral injuries. ${ }^{5}$ The decision to manage first-time dislocations nonoperatively stems from early research that showed no significant difference in recurrence rate or patient-reported outcomes between surgical and conservative management. ${ }^{10-12}$ However, nonoperative management of first-time dislocations in high-risk populations can have recurrence rates that approach $70 \% .{ }^{13}$ Such recurrence is not benign and can result in irreparable cartilage lesions and patellofemoral arthritis. ${ }^{14,15}$ Operative management has been shown to dramatically reduce the risk of recurrence and further chondral damage, and it can result in improved patient-reported outcomes. ${ }^{7,16,17}$ The mainstay of surgical treatment for patients with patellar instability includes repair or reconstruction of the medial patellofemoral ligament. ${ }^{18,19}$ Additionally, concomitant procedures may be performed to address anatomical factors that increase the risk of recurrence, such as lateral retinacular release, trochleoplasty, guided growth, or osteotomy to address genu valgum, and distal extensor mechanism realignment procedures. ${ }^{20-22}$

In addition to providing surgical intervention to the appropriate patients, it is also important that we ensure equity in care. It is well documented that a patient's demographic and socioeconomic status are associated with not only their health-related outcomes, but also their access to care and the offered management options. ${ }^{23-27}$ It is unclear to what degree these disparities may exist in the treatment of patellar instability. The purpose of this study was to determine how patient demographics, socioeconomic status, history of recurrence, and initial point of presentation for healthcare influenced the decision for surgical treatment following a patellar instability episode. We hypothesized that patients who were female, had private insurance, or had a history of recurrent instability would be more likely to undergo surgical intervention for patellar instability. Furthermore, we hypothesized that initial presentation to the emergency department and minority race would be associated with higher costs of care.

\section{Methods}

\section{Data Source}

The data source for this study was outpatient data from the Statewide Planning and Research Cooperative System (SPARCS), an all-payer data system from New York state. Cases from January 1, 2016 to December 31, 2018 were included. This timeframe was chosen to use the newest International Classification of Diseases, 10th revision (ICD-10) diagnosis codes. SPARCS includes data on patient demographics, ICD-10 codes, Current Procedural Terminology (CPT) codes, and charges for services rendered. Data are captured from every ambulatory surgery center, emergency department (ED), and clinic that provide outpatient services in New York state. A unique aspect of this database is the inclusion of both a claim-specific identifier, as well as an anonymized patient identifier that allows for tracking of patients across visits. The "limited" version of SPARCS was used, meaning no direct patient identifiers were available. As such, no approval from an Institutional Review Board was needed.

\section{Case Selection}

All patients 21 years or younger who experienced a patellar dislocation or subluxation were selected using ICD-10 codes S83.001 through S83.096. These diagnosis codes represent lateral, unspecified, and other subluxations and dislocations of the patella. The age cutoff was chosen as the majority of primary patellar instability episodes occur among the pediatric and young adult population. ${ }^{28}$ Further inclusion criteria for dislocation events included presence of CPT codes 99201-99205 (initial office visits), 99211-99215 (established patient office visits), or 99281-99285 (ED visit), as well as a visit date before October 1, 2018, as patients experiencing instability after this date may not have had the opportunity to see an orthopedist. Recurrent instability was defined as having two or more visits with one of the selected ICD-10 codes ending in " $\mathrm{A}$ ", indicating that it was an initial encounter to provide active diagnosis/treatment and not simply a routine follow up visit for a previous instability event. For patients who experienced multiple instability events throughout the course of the study, the first case was used as the index event. Data for patients who subsequently underwent stabilization surgery was then matched using patient identifiers. This was determined using CPT codes 27420 (reconstruction of a dislocating patella), 27422 (Campbell or Roux-Goldthwait type procedure), 27427 (extra-articular knee ligamentous reconstruction), 27418 (tibial tubercle osteotomy), and 27405 (torn collateral ligament/capsule repair). A full description of inclusion criteria is presented in Fig 1. 


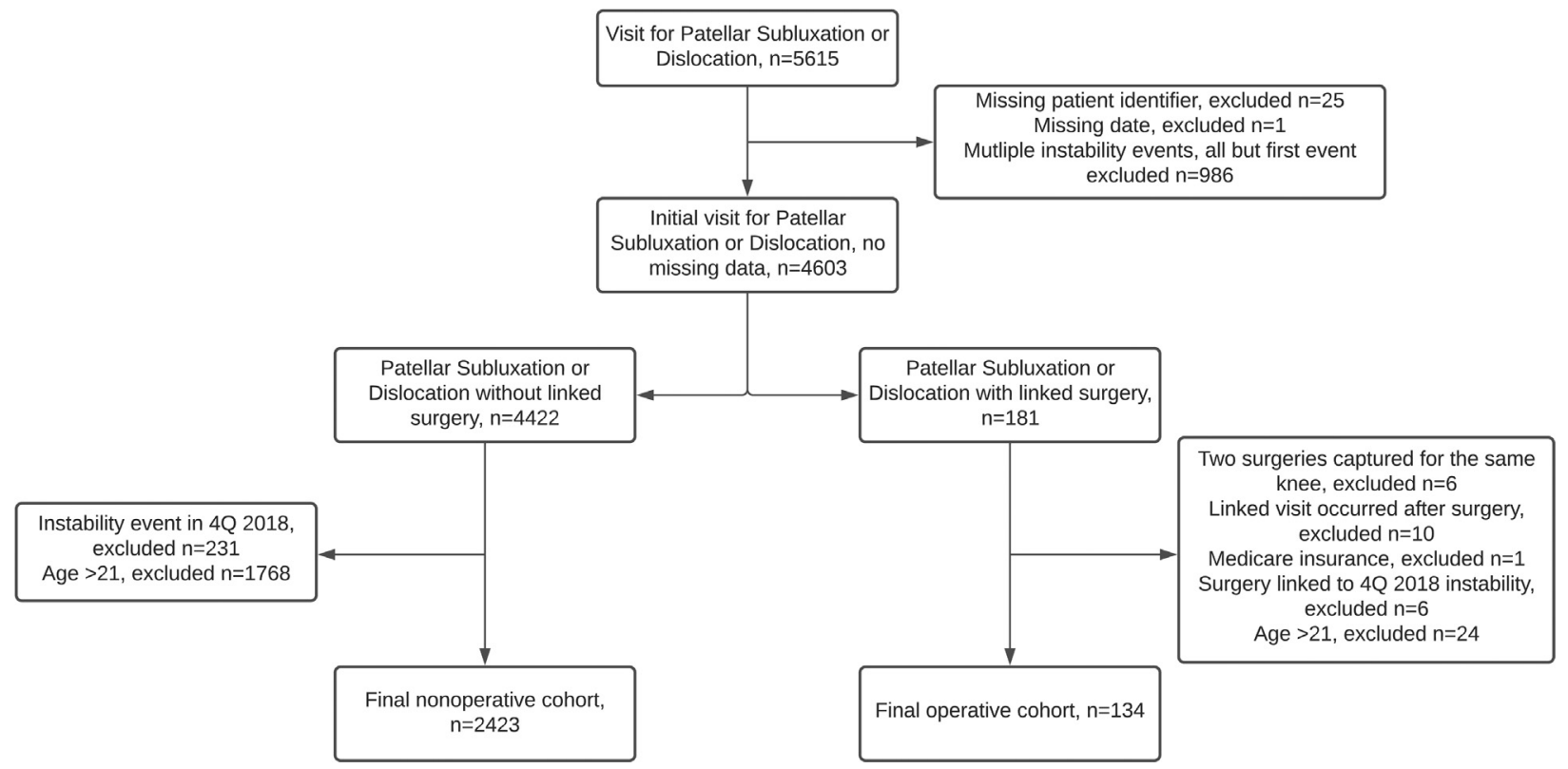

Fig 1. Flowchart of inclusion criteria for patients experiencing patellar instability, Statewide Planning and Research Cooperative System, or SPARCS, database, 2016 to 2018. A total of 2,423 patients were included in the nonoperative cohort, and 134 patients were included in the operative cohort.

\section{Statistical Analysis}

We first performed $\chi^{2}$-analysis to determine the proportion of patients undergoing surgery after an instability event based on several demographic and injury-specific factors, including patient sex, race, insurance, one-time versus repeat instability, type of instability, and site of initial care. Variables that were significant in this analysis were then included in a multivariate binary logistic regression that modeled odds of surgery after experiencing patellar instability. Finally, a multivariate generalized linear model was created to estimate the cost of care surrounding an instability episode, including surgery and concomitant procedures if applicable. The concomitant procedures assessed included chondroplasty (CPT 29877) and lateral release (CPT 27425 and 29873). SPSS V26.0 was used for analysis (IBM Corporation, Armonk, NY).

\section{Results}

After exclusions, there were 2,557 patients who sustained a patella dislocation or subluxation. Of these patients, $134(5.2 \%)$ had a linked surgery, 19 of which were for recurrent instability. There was no difference in mean age between the nonoperative and operative groups ( 15.3 vs 15.2 years; $P=.662$ ). There were 245 patients $(9.6 \%)$ who had recurrent instability. Of these patients, the mean number of instability events occurring during the course of the study was 2.7 . For patients in the operative group, the most common CPT used was $27427(n=60)$, followed by $27422(n=37), 27420$ $(n=26) 27418(n=7)$, and $27405(n=4)$. There were 25 concomitantly performed chondroplasties and 17 lateral releases.
The proportion of patients undergoing surgery after an instability event varied significantly by patient race, insurance, and recurrent instability (Table 1). Hispanic patients had a surgery rate of $2.0 \%$ after an instability event compared with $3.2 \%$ for Black patients and $6.9 \%$ for White patients $(P=.005)$. Privately insured patients had a surgery rate of $5.5 \%$, whereas patients who had no insurance had a surgery rate of $0 \%(P=.009)$. Patients with recurrent instability had a surgery rate of $7.8 \%$ compared with $4.5 \%$ for patients with one-time instability $(P=.023)$. There was no difference in rate of surgery by patient sex, subluxation versus dislocation, or site of initial care.

Patient sex, race, insurance, recurrent instability, and instability type were also associated with ED utilization (Table 2). Female patients were less likely to use the ED for initial care than male patients (Odds Ratio [OR] $.691, P=.001)$. White patients were the most likely to use the ED, whereas Black patients (OR .371, $P<.001$ ) and patients of another race (OR .320, $P<.001)$ were significantly less likely to present to the ED for initial care. Compared with privately insured patients, patients with Medicaid were less likely to use the ED (OR $.640, P<.001)$. Patients without insurance were more likely to present to the ED (OR 1.881, $P=.016$ ). Instability recurrence was also a factor, as only $33.1 \%$ of patients with recurrent instability used the ED compared with $87.6 \%$ of first-time dislocations (OR $.070, P<.001)$. Finally, $67.6 \%$ of subluxations resulted in an ED visit compared to $85.0 \%$ of dislocations (OR 2.708, $P<.001)$.

Both patient race and recurrent instability were found to be independent predictors of surgery under a binary 
Table 1. Percentage of Patients Undergoing Surgical Stabilization after an Episode of Patellar Instability and $\chi^{2}$-Analysis, 2016-2018

\begin{tabular}{|c|c|c|c|c|}
\hline \multirow[b]{2}{*}{ Variable } & \multirow[b]{2}{*}{ Group } & \multicolumn{3}{|c|}{$\begin{array}{c}\text { Percent } \\
\text { Undergoing }\end{array}$} \\
\hline & & $N$ & Surgery & $P$ Value \\
\hline \multirow[t]{2}{*}{ Sex } & Male & 1173 & $4.9 \%$ & 0.426 \\
\hline & Female & 1384 & $5.6 \%$ & \\
\hline \multirow[t]{6}{*}{ Race } & White & 1300 & $6.9 \%$ & $0.005^{*}$ \\
\hline & Black & 432 & $3.2 \%$ & \\
\hline & Hispanic & 98 & $2.0 \%$ & \\
\hline & Asian & 119 & $2.5 \%$ & \\
\hline & Native American & 14 & $7.1 \%$ & \\
\hline & Other & 594 & $4.0 \%$ & \\
\hline \multirow[t]{4}{*}{ Insurance } & Private Insurance & 1705 & $5.9 \%$ & $0.009^{*}$ \\
\hline & Medicaid & 609 & $4.9 \%$ & \\
\hline & Self-Pay & 177 & $0.0 \%$ & \\
\hline & Other & 66 & $4.5 \%$ & \\
\hline \multirow[t]{2}{*}{ Recurrent instability } & No & 2312 & $5.0 \%$ & 0.023 \\
\hline & Yes & 245 & $7.8 \%$ & \\
\hline \multirow[t]{2}{*}{ Type of Instability } & Subluxation & 380 & $4.7 \%$ & 0.633 \\
\hline & Dislocation & 2177 & $5.3 \%$ & \\
\hline \multirow[t]{2}{*}{ Site of initial care } & ED & 2106 & $5.2 \%$ & 0.932 \\
\hline & Office Visit & 451 & $5.3 \%$ & \\
\hline
\end{tabular}

ED, Emergency Department.

${ }^{*} P$ value denotes overall significance.

logistic regression (Table 3). Patients with recurrent instability had 1.875 times the odds of undergoing stabilization surgery than patients with one-time instability $(P=.017)$. Compared to White patients, Black patients had 0.428 times the odds of surgery $(P=$ $0.004)$. Hispanic patients had 0.268 times the odds of surgery compared to White patients, but this difference was not significant $(P=.069)$.

We found several independent drivers of charges for care after patellar instability (Table 4). Compared with using the ED for care, an initial visit to an outpatient office was associated with $\$ 1,994$ less in charges $(P<$ $.001)$. Black patients had $\$ 566$ more charges than
White patients $(P=.009)$. Compared with nonoperative treatment, surgical treatment had significantly higher charges. Specifically, CPT 27422 added $\$ 19,198$ $(P<.001)$, CPT 27420 added $\$ 24,443(P<.001)$, CPT 27427 added $\$ 25,259(P<.001)$, CPT 27405 added $\$ 13,124(P<.001)$, and CPT 27418 added $\$ 10,749$ in charges $(P<.001)$. For patients undergoing surgery, a concomitant chondroplasty added $\$ 6,116(P<.001)$, and lateral release added $\$ 9,106(P<.001)$.

\section{Discussion}

In the current study, 5.2\% of patients required surgical management for their patellar instability. Independent predictors of surgery following a patellar instability event included patient race and recurrent instability. We also determined that drivers of increased charges included an initial emergency room consultation, race, surgical management, and the performance of concomitant procedures. These results support our hypothesis. Although it is expected that recurrent instability would be a predictor of surgical intervention, it is not clear why race would be a factor.

The relationship between race and surgical management of orthopaedic injuries has been explored in several facets. Navarro et al. identified differences in sex, age, mechanism of injury, and surgeon training when comparing various racial groups undergoing anterior ligament reconstruction. ${ }^{29}$ In rotator cuff repair surgery, Hispanic patients were shown to be significantly less likely to receive surgical care from a high-volume surgeon or at a high-volume facility. ${ }^{24}$ With regard to patellar instability, Waterman et al. observed significantly greater incidence rates of patellar dislocation in Black and White patients compared with patients of Hispanic race; however, these authors did not consider factors affecting surgery after dislocation. ${ }^{2}$ Our findings demonstrate that Black patients have

Table 2. Percentage of Patients Visiting the Emergency Department for Patellar Instability with Odds Ratios, 2016-2018

\begin{tabular}{|c|c|c|c|c|}
\hline Variable & Group & Percent Visiting ED & Odds Ratio $(95 \% \mathrm{CI})$ & $P$ Value \\
\hline \multirow[t]{2}{*}{$\overline{\operatorname{Sex}}$} & Male & $85.3 \%$ & Reference & \\
\hline & Female & $80.0 \%$ & $.691(0.561$ to .851$)$ & .001 \\
\hline \multirow[t]{6}{*}{ Race } & White & $89.0 \%$ & Reference & \\
\hline & Black & $75.0 \%$ & $.371(.281$ to .490$)$ & $<.001$ \\
\hline & Hispanic & $84.7 \%$ & $.684(.384$ to 1.217$)$ & .197 \\
\hline & Asian & $83.2 \%$ & $.612(.367$ to 1.020$)$ & .059 \\
\hline & Native American & $85.7 \%$ & $.742(.164$ to 3.347$)$ & .697 \\
\hline & Other & $72.7 \%$ & $.320(.257$ to 0.423$)$ & $<.0001$ \\
\hline \multirow[t]{4}{*}{ Insurance } & Private Insurance & $83.3 \%$ & Reference & \\
\hline & Medicaid & $76.2 \%$ & $.640(.510$ to 0.802$)$ & $<.001$ \\
\hline & None & $90.4 \%$ & $1.881(1.123$ to 3.151$)$ & .016 \\
\hline & Other & $93.9 \%$ & $3.098(1.118$ to 8.583$)$ & .030 \\
\hline \multirow[t]{2}{*}{ Instability Recurrence } & One-time & $87.6 \%$ & Reference & \\
\hline & Recurrent & $33.1 \%$ & $.070(.052$ to .094$)$ & $<.001$ \\
\hline \multirow[t]{2}{*}{ Instability type } & Subluxation & $67.6 \%$ & Reference & \\
\hline & Dislocation & $85.0 \%$ & $2.708(2.119$ to 3.459$)$ & $<.001$ \\
\hline
\end{tabular}

CI: confidence interval; ED, Emergency Department. 
Table 3. Binary Logistic Regression of Odds of Surgery after a Patellar Instability Event

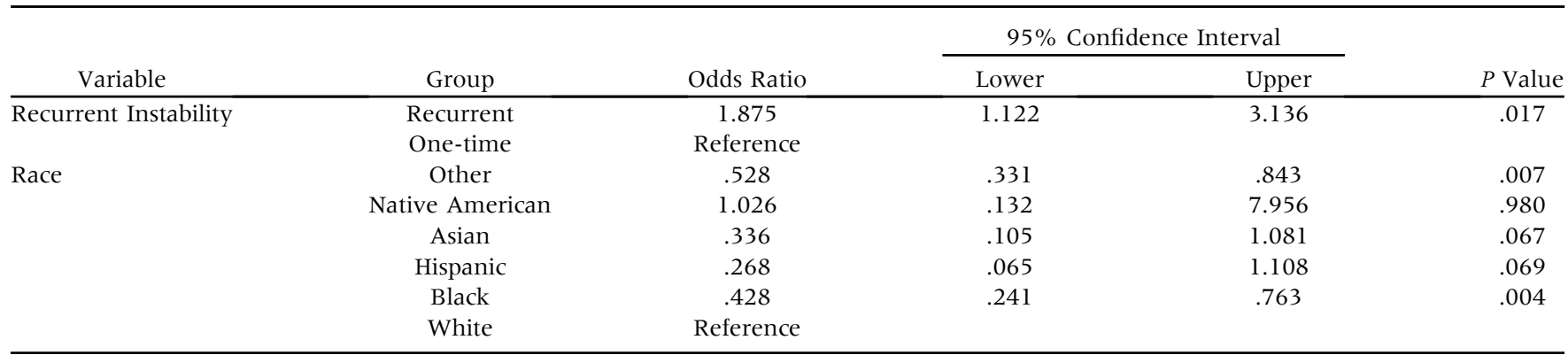

Variables that were assessed but were not significant predictors: sex, age, insurance, and subluxation vs dislocation.

significantly decreased odds of undergoing patellar stabilization surgery compared with white patients (OR $.428, P=.004)$. These numbers suggest there may be racial disparity in the operative management of patellar instability, and further research into understanding the reason for such differences would be beneficial.

Our findings also show that race may be a factor affecting the cost of care for patellar instability patients. For example, our multivariable regression revealed that the charges associated with care for Black patients was significantly higher than that for White patients $(+\$ 566, P=.009)$, even when adjusting for other variables, such as insurance, site of initial care, and operative versus nonoperative treatment. Hispanic and Asian patients followed a similar trend at $\$ 458$ and $\$ 491$ more charges on average, although these values did not reach the level of statistical significance $(P=$ .222 and $P=.252$, respectively). This trend has been noted previously in total joint arthroplasty surgery, with Black patients having a $\$ 440$ increase in the cost of care episodes, even when controlling for possible confounders. ${ }^{30}$ These authors implicated increased rates of home health and rehabilitation as a cause for increased costs in this population, which would not be expected to be an issue with an outpatient procedure addressing patellar instability. Moreover, it was found that Black patients were less likely to use the ED for care, which itself is associated with higher costs, so the cost disparity may be related to charges incurred during the initial episodes of care. Although we do not have data on what specific factors lead to increased costs among black patients, future studies may investigate specific cost drivers not accounted for here, such as PACU time, durable medical equipment, and physical therapy usage. Such trends are important for surgeons, insurers, and policymakers alike to appreciate as equitable and affordable health care costs are sought.

The charges associated with undergoing surgical intervention for patellar instability has a wide range, with our study identifying average surgical charges starting between $\$ 10,000$ and $\$ 25,000$ depending on the CPT code utilized (Table 4). Other important factors

Table 4. Charges Associated with Patellar Instability

\begin{tabular}{|c|c|c|c|c|c|}
\hline Variable & Group & Cost & \multicolumn{2}{|c|}{ 95\% Confidence Interval } & $P$ Value \\
\hline \multirow{2}{*}{ Site of initial care } & Intercept & $\$ 2,235$ & $\$ 2,005$ & $\$ 2,464$ & $<.001$ \\
\hline & ED visit & Reference & & & \\
\hline \multirow[t]{4}{*}{ Race } & Other & $\$ 385$ & $-\$ 13$ & $\$ 784$ & .051 \\
\hline & Native American & $\$ 848$ & $-\$ 1,284$ & $\$ 2,979$ & .827 \\
\hline & Asian & $\$ 491$ & $-\$ 269$ & $\$ 1,250$ & .252 \\
\hline & White & Reference & & & \\
\hline \multirow[t]{4}{*}{ Treatment } & Tibial tubercle osteotomy (CPT 27418) & $\$ 10,749$ & $\$ 7,396$ & $\$ 14,103$ & $<.001$ \\
\hline & Torn collateral ligament/capsule repair (CPT 27405) & $\$ 13,124$ & $\$ 9,131$ & $\$ 17,117$ & \\
\hline & Extra-articular ligamentous reconstruction (CPT 27427) & $\$ 25,259$ & $\$ 24,144$ & $\$ 26,375$ & \\
\hline & $\begin{array}{l}\text { Extensor realignment - Campbell/Goldthwait procedure } \\
\text { (CPT 27422) }\end{array}$ & $\$ 19,198$ & $\$ 17,754$ & $\$ 20,643$ & \\
\hline
\end{tabular}

Variables assessed but that were not significant predictors: sex, insurance, age, subluxation vs dislocation, and recurrent instability.

ED, Emergency Department. 
identified that may affect cost include the additional coding of concomitant chondroplasty or lateral release. These procedures may be an adjunct to MPFL reconstruction, which has become commonplace in the treatment of patellar instability, depending on any additional pathology, which must be addressed. ${ }^{31}$ of note, lateral release charges were nearly $\$ 10,000$ more than the standard MPFL reconstruction, resulting in the greatest increase in cost to surgical management of patellar instability in our cohort. Given the currently debated indications for lateral release, these data may help inform decision making around cost-conscious care. $^{32}$

Nwachukwu et al. performed a cost-effectiveness analysis of first-time patellar dislocations in adolescents, finding that nonoperative treatment has been shown to be the least costly but also least effective procedure, with a cost of $\$ 7,300$ and lower qualityadjusted life years (QALY) than immediate or delayed surgery. ${ }^{33}$ They concluded that immediate surgery and delayed surgery were both cost-effective options; however, their model was sensitive to the probability of a recurrent instability episode following initial nonoperative treatment. Our results demonstrate higher rates of surgical management in patients having multiple instability events compared with a single event. This suggests that surgeons continue to attempt nonoperative management after first-time instability events, which is in line with current literature, suggesting good outcomes with nonoperative management in initial dislocators. ${ }^{34}$ There was no difference in surgery rates between patients who had subluxations versus dislocations, indicating that the recurrence of instability is a more important factor than degree of initial instability. It should be noted that the $9.6 \%$ rate of recurrence in our study is much lower than the $>30 \%$ rate that has been reported in recent systematic reviews. ${ }^{35,36}$ This is likely because of the shorter time period covered by this study, as the median time from instability event to the end of the study was 16 months.

Another factor affecting the cost of a care is an initial emergency room-based consultation for patellar instability. Anderson et al. reviewed a series of patients with musculoskeletal complaints evaluated in an orthopaedic urgent care center versus the emergency room setting, establishing a $\$ 8,150$ average cost for patients evaluated in a hospital emergency room, compared with only $\$ 461$ for the urgent care setting. ${ }^{37}$ In 2017 , the state of New York permitted emergency medical technicians (EMTs) to perform patella relocations, given the general ease of reduction and favorable risk-benefit profile. ${ }^{38}$ Lord et al. found that $90 \%$ patellar dislocations were successfully reduced by New York EMT providers, but ultimately $77.4 \%$ of patients received transport to the hospital to be evaluated in the emergency room. ${ }^{38}$ This large percentage is similar to the
$82 \%$ of patients initially treated in the emergency room for their patellar instability event, as determined by our study (Table 1). Patient education and prior experience with PI may be an especially important factor given the low ED utilization among patients with recurrent instability in our study. Less than one-third of these patients presented to the ED for care, compared with $87.6 \%$ of first-time dislocators. As these patients had experienced instability before, they may have been more inclined to avoid the ED and use urgent care centers or orthopaedic practices.

Health insurance status is a well-known barrier to the full scope of medical care in the United States, and has been shown to affect surgical management, as well as outcomes after orthopaedic and spine surgery. ${ }^{39,40,41}$ Our findings implicate insurance status as another factor affecting the rate of surgical management of patellar instability. There were zero uninsured patients who underwent surgical intervention in our cohort, while privately insured patients had the highest rate of surgical management at 5.9\% $(P<.001)$ (Table 1). Patients who do not have insurance coverage may be unable to afford the high costs of surgery out-of-pocket, thus contributing to an important barrier in the access to surgical care. Unfortunately, these uninsured patients were also more likely to use the ED for care, which is an additional cost burden. Similar findings have previously been observed in other studies. For example, Daly et al. found that patients with health insurance have a significantly higher rate of surgical intervention following spine fractures, regardless of evidence of spinal cord injury. ${ }^{40}$ Gundle and Ramappa showed that self-pay patients have a significantly lower chance of undergoing surgery following a meniscal tear. ${ }^{42}$ As the United States continues to evolve with respect to health insurance policy, it is essential to recognize and appreciate the disparities observed in surgical care in the underinsured and seek to ensure their access to care to optimize patient outcomes.

\section{Limitations}

This study contains several inherent limitations. First, the nature of all database studies reflects limitations seen in retrospective investigations, as well as requires accurate coding to ensure adequate data and valid results. As with any database study, we cannot be sure what the coding accuracy is because we cannot access the patient notes, and there is always the risk that billing practices may not accurately depict subluxation versus dislocation or initial versus recurrent instability. Patellar instability can range from simple subluxation to dislocation, but dislocation events may also be associated with concomitant injuries, such as multiligamentous knee injuries and displaced osteochondral fragments. ${ }^{34}$ We were unable to specifically consider individual, patient-specific injury patterns or patient 
morphologies given the large, retrospective, nature of our study; however, we limited patients to those who only had patellar instability-specific diagnosis codes. Although we excluded instability events from the fourth quarter of 2018, it is possible that patients experiencing instability before this were managed operatively after December 31, 2018, giving a surgery rate that is lower than what may actually exist. Finally, our data are limited geographically to New York state via use of the SPARCS database. Therefore, national and global trends cannot be directly considered, possibly limiting appropriate extrapolation to other areas.

\section{Conclusion}

Patients with recurrent instability had higher odds of surgery, while Black and uninsured patients had lower odds of surgery. ED visits were associated with significantly higher charges compared to office visits, and Black patients had higher charges than White patients. Minority and uninsured patients may face barriers in access to orthopedic care.

\section{References}

1. Colvin AC, West RV. Patellar instability. J Bone Jt Surg Ser A 2008;90:2751-2762. doi:10.2106/JBJS.H.00211.

2. Waterman BR, Belmont PJ, Owens BD. Patellar dislocation in the United States: Role of sex, age, race, and athletic participation. J Knee Surg 2011;25:51-58. doi:10. 1055/s-0031-1286199.

3. Mitchell J, Magnussen RA, Collins CL, et al. Epidemiology of patellofemoral instability injuries among high school athletes in the United States. Am J Sports Med 2015;43: 1676-1682. doi:10.1177/0363546515577786.

4. Trojan JD, Treloar JA, Smith CM, Kraeutler MJ, Mulcahey MK. Epidemiological patterns of patellofemoral injuries in collegiate athletes in the United States From 2009 to 2014. Orthop J Sport Med 2019;7. doi:10.1177/ 2325967119840712:2325967119840712.

5. Sillanpää PJ, Mäenpää HM. First-time patellar dislocation: Surgery or conservative treatment? Sports Med Arthrosc 2012;20:128-135. doi:10.1097/JSA.0b013e318256bbe5.

6. Erickson BJ, Mascarenhas R, Sayegh ET, et al. Does operative treatment of first-time patellar dislocations lead to increased patellofemoral stability? A systematic review of overlapping meta-analyses. Arthroscopy 2015;31: 1207-1215. doi:10.1016/j.arthro.2014.11.040.

7. Gao B, Dwivedi S, Fabricant PD, Cruz AI. Patterns in outcomes reporting of operatively managed pediatric patellofemoral instability: A systematic review and metaanalysis. Am J Sports Med 2019;47:1516-1524. doi:10. $1177 / 0363546518765152$.

8. Arshi A, Cohen JR, Wang JC, Hame SL, McAllister DR, Jones KJ. Operative management of patellar instability in the United States: An evaluation of national practice patterns, surgical trends, and complications. Orthop J Sport Med 2016;4: 232596711666287. doi:10.1177/2325967116662873.

9. Liu JN, Steinhaus ME, Kalbian IL, et al. Patellar instability management: A survey of the international patellofemoral study group. Am J Sports Med 2018;46:3299-3306. doi:10. $1177 / 0363546517732045$.

10. Nikku R, Nietosvaara Y, Aalto K, Kallio PE. Operative treatment of primary patellar dislocation does not improve medium-term outcome: A 7-year follow-up report and risk analysis of 127 randomized patients. Acta Orthop 2005;76: 699-704. doi:10.1080/17453670510041790.

1 1. Sillanpää PJ, mattila V M, Mäenpää H, Kiuru M, Visuri T, Pihlajamäki H. Treatment with and without initial stabilizing surgery for primary traumatic patellar dislocation: A prospective randomized study. J Bone Jt Surg Am 2009;91: 263-273. doi:10.2106/JBJS.G.01449.

12. Smith TO, Donell S, Song F, Hing CB. Surgical versus non-surgical interventions for treating patellar dislocation. Cochrane Database Syst Rev 2015;(2):CD008106. doi: 10.1002/14651858.CD008106.pub3.

13. Lewallen LW, McIntosh AL, Dahm DL. Predictors of recurrent instability after acute patellofemoral dislocation in pediatric and adolescent patients. Am J Sports Med 2013;41:575-581. doi:10.1177/0363546512472873.

14. Nomura E, Inoue M, Kobayashi S. Long-term follow-up and knee osteoarthritis change after medial patellofemoral ligament reconstruction for recurrent patellar dislocation. Am J Sports Med 2007;35:1851-1858. doi:10. $1177 / 0363546507306161$.

15. Nomura E, Inoue M, Kurimura M. Chondral and osteochondral injuries associated with acute patellar dislocation. Arthroscopy 2003;19:717-721. doi:10.1016/S07498063(03)00401-8.

16. Nwachukwu BU, So C, Schairer WW, Green DW, Dodwell ER. Surgical versus conservative management of acute patellar dislocation in children and adolescents: A systematic review. Knee Surg Sport Traumatol Arthrosc 2016;24:760-767. doi:10.1007/s00167-015-3948-2.

17. Bitar AC, Demange MK, D’Elia CO, Camanho GL. Traumatic patellar dislocation: Nonoperative treatment compared with MPFL reconstruction using patellar tendon. Am J Sports Med 2012;40:114-122. doi:10.1177/ 0363546511423742.

18. Hautamaa PV., Fithian DC, Kaufman KR, Daniel DM, Pohlmeyer AM. Medial soft tissue restraints in lateral pateliar instability and repair. Clin Orthop Relat Res 1998;(349): 174-182. doi:10.1097/00003086-199804000-00021.

19. Weber AE, Nathani A, Dines JS, et al. An algorithmic approach to the management of recurrent lateral patellar dislocation. J Bone Joint Surg Am 2016;98:417-427. doi:10. $2106 /$ JBJS.O.00354.

20. Marsh JS, Daigneault JP, Sethi P, Polzhofer GK. Treatment of recurrent patellar instability with a modification of the Roux-Goldthwait technique. J Pediatr Orthop 2006;26: 461-465. doi:10.1097/01.bpo.0000217711.34492.48.

21. Lattermann C, Toth J, Bach BR. The role of lateral retinacular release in the treatment of patellar instability. Sports Med Arthrosc 2007;15:57-60. doi:10.1097/JSA. 0b013e318042af30.

22. Tan SHS, Tan LYH, Lim AKS, Hui JH. Hemiepiphysiodesis is a potentially effective surgical management for skeletally immature patients with patellofemoral instability associated with isolated genu valgum. Knee Surg Sport Traumatol Arthrosc 2019;27:845-849. doi:10.1007/s00167018-5127-8. 
23. Andrulis DP. Access to care is the centerpiece in the elimination of socioeconomic disparities in health. Ann Intern Med 1998;129:412-416. doi:10.7326/0003-4819. 129-5-199809010-00012.

24. Li L, Bokshan SL, Mehta SR, Owens BD. Disparities in cost and access by caseload for arthroscopic rotator cuff repair: An analysis of 18,616 cases. Orthop J Sport Med 2019;7. doi:10.1177/2325967119850503:2325967119850503.

25. Labrum JT 4th, Paziuk T, Rihn TC, et al. Does Medicaid insurance confer adequate access to adult orthopaedic care in the era of the patient protection and Affordable Care Act? Clin Orthop Relat Res 2017;475:1527-1536. doi: 10.1007/s1 1999-017-5263-3.

26. Skaggs DL, Lehmann CL, Rice C, et al. Access to orthopaedic care for children with medicaid versus private insurance: results of a national survey. J Pediatr Orthop 2006;26:400-404. doi:10.1097/01.bpo. 0000217715.87857 .24

27. Wiznia DH, Nwachuku E, Roth A, et al. The influence of medical insurance on patient access to orthopaedic surgery sports medicine appointments under the Affordable Care Act. Orthop J Sport Med 2017;5:2325967117714140. doi:10.1177/2325967117714140.

28. Grimm NL, Levy BJ, Jimenez AE, Crepeau AE, Lee Pace J. Traumatic patellar dislocations in childhood and adolescents. Orthop Clin North Am 2020;51:481-491. doi:10. 1016/j.ocl.2020.06.005.

29. Navarro RA, Prentice HA, Inacio MCS, Wyatt $R$, Maletis GB. The association between race/ethnicity and revision following ACL reconstruction in a universally insured cohort. J Bone Jt Surg 2019;101:1546-1553. doi: 10.2106/JBJS.18.01408.

30. Chisari E, Grosso MJ, Kozaily E, Nelson CL, Sherman MB, Courtney PM. High bundled payment costs following total hip and knee arthroplasty in African Americans. J Arthroplasty 2021;36:19-23. doi:10.1016/j.arth.2020.07.053.

31. Diduch DR, Kandil A, Burrus MT. Lateral patellar instability in the skeletally mature patient: Evaluation and surgical management. J Am Acad Orthop Surg 2018;26: 429-439. doi:10.5435/JAAOS-D-16-00052.

32. Migliorini F, Maffulli N, Eschweiler J, Quack V, Tingart M, Driessen A. Lateral retinacular release combined with MPFL reconstruction for patellofemoral instability: A systematic review. Arch Orthop Trauma Surg 2021;141: 283-292. doi:10.1007/s00402-020-03689-9.

33. Nwachukwu BU, So C, Schairer WW, et al. Economic decision model for first-time traumatic patellar dislocations in adolescents. Am J Sports Med 2017;45:2267-2275. doi: $10.1177 / 0363546517703347$.

34. Jain NP, Khan N, Fithian DC. A treatment algorithm for primary patellar dislocations. Sports Health 2011;3: 170-174. doi:10.1177/1941738111399237.

35. Huntington LS, Webster KE, Devitt BM, Scanlon JP, Feller JA. Factors associated with an increased risk of recurrence after a first-time patellar dislocation: A systematic review and meta-analysis. Am J Sports Med 2020;48:2552-2562. doi:10.1177/0363546519888467.

36. Longo UG, Ciuffreda M, Locher J, Berton A, Salvatore G, Denaro V. Treatment of primary acute patellar dislocation: Systematic review and quantitative synthesis of the literature. Clin J Sport Med Off J Can Acad Sport Med 2017;27: 511-523. doi:10.1097/JSM.0000000000000410.

37. Anderson TJ, Althausen PL. The role of dedicated musculoskeletal urgent care centers in reducing cost and improving access to orthopaedic care. J Orthop Trauma 2016;30:S3-S6. doi:10.1097/BOT.0000000000000712.

38. Lord S, Brodell J, Lenhardt H, Dailey M, Cushman J. Implementation of a prehospital patella dislocation reduction protocol. Prehospital Emerg Care 2020;24: 800-803. doi:10.1080/10903127.2019.1704322.

39. Li X, Veltre DR, Cusano A, et al. Insurance status affects postoperative morbidity and complication rate after shoulder arthroplasty. J Shoulder Elb Surg 2017;26: 1423-1431. doi:10.1016/j.jse.2016.12.071.

40. Daly MC, Patel MS, Bhatia NN, Bederman SS. The influence of insurance status on the surgical treatment of acute spinal fractures. Spine (Phila $\mathrm{Pa}$ 1976) 2016;41: E37-E45. doi:10.1097/BRS.0000000000001177.

41. Dasenbrock HH, Wolinsky J-P, Sciubba DM, Witham TF, Gokaslan ZL, Bydon A. The impact of insurance status on outcomes after surgery for spinal metastases. Cancer 2012;118:4833-4841. doi:10.1002/cncr.27388.

42. Gundle KR, McGlaston TJ, Ramappa AJ. Effect of insurance status on the rate of surgery following a meniscal tear. J Bone Jt Surg Am 2010;92:2452-2456. doi:10.2106/ JBJS.I.01369. 